\title{
The Dunaliella salina organelle genomes: large sequences, inflated with intronic and intergenic DNA
}

\author{
David Roy Smith*1, Robert W Lee1, John C Cushman², Jon K Magnuson³, Duc Tran ${ }^{4}$ and Jürgen EW Polle4
}

\begin{abstract}
Background: Dunaliella salina Teodoresco, a unicellular, halophilic green alga belonging to the Chlorophyceae, is among the most industrially important microalgae. This is because $D$. salina can produce massive amounts of $\beta$ carotene, which can be collected for commercial purposes, and because of its potential as a feedstock for biofuels production. Although the biochemistry and physiology of D. salina have been studied in great detail, virtually nothing is known about the genomes it carries, especially those within its mitochondrion and plastid. This study presents the complete mitochondrial and plastid genome sequences of $D$. salina and compares them with those of the model green algae Chlamydomonas reinhardtii and Volvox carteri.
\end{abstract}

Results: The D. salina organelle genomes are large, circular-mapping molecules with $\sim 60 \%$ noncoding DNA, placing them among the most inflated organelle DNAs sampled from the Chlorophyta. In fact, the D. salina plastid genome, at $269 \mathrm{~kb}$, is the largest complete plastid DNA (ptDNA) sequence currently deposited in GenBank, and both the mitochondrial and plastid genomes have unprecedentedly high intron densities for organelle DNA: 1.5 and 0.4 introns per gene, respectively. Moreover, what appear to be the relics of genes, introns, and intronic open reading frames are found scattered throughout the intergenic ptDNA regions -- a trait without parallel in other characterized organelle genomes and one that gives insight into the mechanisms and modes of expansion of the D. salina ptDNA.

Conclusions: These findings confirm the notion that chlamydomonadalean algae have some of the most extreme organelle genomes of all eukaryotes. They also suggest that the events giving rise to the expanded ptDNA architecture of D. salina and other Chlamydomonadales may have occurred early in the evolution of this lineage. Although interesting from a genome evolution standpoint, the D. salina organelle DNA sequences will aid in the development of a viable plastid transformation system for this model alga, and they will complement the forthcoming $D$. salina nuclear genome sequence, placing $D$. salina in a group of a select few photosynthetic eukaryotes for which complete genome sequences from all three genetic compartments are available.

\section{Background}

Dunaliella salina Teodoresco [1] is one of the best-studied unicellular green algae [2-4]. This is not only because D. salina is halotolerant, thriving in extreme saline environments [3], but also because it can produce large quantities of $\beta$-carotene (up to $10 \%$ of the cell's dry weight) in lipid globules located within the chloroplast $[5,6]$. These traits make $D$. salina a model organism for investigating the evolution of salt adaptation [2] and an attractive "cell

* Correspondence: smithdr@dal.ca

1 Department of Biology, Dalhousie University, Halifax, NS, B3H 4J1, Canada Full list of author information is available at the end of the article factory" for the commercial production of $\beta$-carotene $[7,8]$. Although a great deal is known about the physiology and biochemistry of $D$. salina $[9,10]$, very little is known about the genomes it carries, especially those within its organelles. Until now, nothing was known about the size, conformation, or gene complement of either the mitochondrial or plastid genomes of $D$. salina (or those of any other Dunaliella species) even though the sequences of these genomes are essential to the development of new $D$. salina technologies, such as a viable plastid transformation system [11-16]. 
Research on green-algal organelle genomes has led to significant advancements in genetic engineering. The first stable transformation of a plastid genome was achieved in 1988 using the unicellular green alga Chlamydomonas reinhardtii [17] and, soon after, the first example of recombinant protein expression in a plastid was also achieved using C. reinhardtii [18]. Since then, many techniques for plastid engineering have been first developed for green algae and then adapted for use in land plants [19]. Given the relatively close evolutionary proximity of C. reinhardtii and D. salina [20], it is reasonable to assume that many of the technologies for $C$. reinhardtii plastid transformation might be transferable to $D$. salina. Various groups have attempted to transform $D$. salina [21]; however, a lack of plastid-genome sequence data has prevented successful plastid transformation. Therefore, the first step in developing an efficient and reliable plastid transformation system for $D$. salina is to sequence its organelle genomes.

D. salina is an attractive alga for organelle genome research and plastome engineering for a variety of reasons: i) various strains and geographical isolates of $D$. salina are readily available from algal culture collections around the world; ii) D. salina is relatively easy to grow and maintain -- it is one of the few microalgae that are being cultivated currently on a large scale; iii) $D$. salina lacks a rigid cell wall, facilitating organelle DNA extraction; iv) D. salina is unicellular, with only a single plastid, making it easier, as compared with multicellular species, to develop homoplasmic lines of plastid transformants; and v) being a close relative of the model green algae $C$. reinhardtii and Volvox carteri, means D. salina is an ideal species for comparative plant studies, especially comparative genomics, because the United States Department of Energy Joint Genome Institute (DOE JGI) is sequencing, or has sequenced, the $C$. reinhardtii, $V$. carteri, and $D$. salina nuclear genomes.

In 2006, the DOE JGI began sequencing the $D$. salina strain CCAP (Culture Collection of Algae and Protozoa) $19 / 18$ nuclear genome, which is approximately 300 megabases $(\mathrm{Mb})$ in length [[22]; DOE JGI, personal communication]. D. salina was selected for genome sequencing because of its potential as a feedstock producer for biofuels production [23] and its model status for studying saline adaptation. All of the $D$. salina whole genome shotgun sequencing (WGS) trace files that the DOE JGI produced are publicly available at the GenBank Trace Archive [24]; soon a complete assembly of the $D$. salina nuclear DNA (nucDNA) will be made public. The fact that $D$. salina is the third chlamydomonadalean alga for which there is a genome sequencing project, reaffirms that the Chlamydomonadales are emerging as the one of foremost lineages for comparative genomics.
The taxonomic position of the Dunaliella genus is still under debate [25]; however, it is often placed within the Chlamydomonadales (Chlorophyceae, Chlorophyta). For the purpose of this study, our definition of the Chlamydomonadales follows that of Lewis and McCourt [26], which includes the Dunaliella genus, and is equivalent to both the basal-bodies-clockwise group (CW group) [26] and the Volvocales sensu Nakada et al. [20]. Notably, some strains of $D$. salina were incorrectly identified in the past, which resulted in the deposition of inaccurately labeled DNA sequence data in public databases. Moreover, there exists a debate regarding the delineation of the species D. salina Teod. and Dunaliella bardawil Avron et Ben-Amotz [3,25]. According to Borowitzka and Borowitzka [27] and Borowitzka and Siva [28], the species $D$. bardawil is a nomen nudum; however, the name $D$. bardawil is still in use. Given the above issues, one should exercise great caution when using DNA sequence data in public databases that are said to have originated from $D$. salina.

As of November 1, 2009, complete and almost complete organelle DNA sequences are available for eight chlamydomonadalean algae, amounting to two plastid and eight mitochondrial genome sequences [29-38]; moreover, comprehensive genetic maps and limited sequence data are available for the plastid genomes of an additional four taxa [39-41]. The general features of these organelle genome data, as well as the species from which they are derived, are summarized in Table 1. Many of the available chlamydomonadalean organelle genome sequences are atypical in one way or another, having extreme sizes (e.g., large and expanded or highly compact $[30,37])$, unusual conformations (e.g., linear or linear fragmented $[33,36,42])$, and/or severely biased nucleotide composition (e.g., GC- or AT-rich [37,43]). Furthermore, there can be extensive size, conformational and/or compositional differences among the organelle genomes of closely related chlamydomonadalean species $[36,41,43,44]$. This wide assortment of genome architectures makes the Chlamydomonadales an ideal lineage for studying genome evolution $[35,38,45,46]$.

Most of our knowledge of chlamydomonadalean organelle genomes comes from species within the Reinhardtinia clade (defined by Nakada et al. [20]). Indeed, six of the eight chlamydomonadalean algae for which significant organelle DNA sequence data are available come from this clade (Table 1), including C. reinhardtii and $V$. carteri. Given that the Reinhardtinia clade contains only a small fraction of the species diversity within the Chlamydomonadales, it would be intriguing to explore the organelle genomes of algae from other chlamydomonadalean clades. It would be particularly interesting to see if chlamydomonadalean algae from outside the 
Table 1: Available organelle-genome data for chlamydomonadalean algae.

\begin{tabular}{|c|c|c|c|c|c|c|c|}
\hline Genus and species & Clade & $\begin{array}{c}\text { Mapping } \\
\text { conformation }\end{array}$ & Size (kb) & $\begin{array}{c}\% \\
\text { coding }\end{array}$ & \%GC & $\begin{array}{l}\text { GenBank } \\
\text { Accession }\end{array}$ & Reference \\
\hline \multicolumn{8}{|l|}{ MITOCHONDRIAL GENOMES } \\
\hline Chlamydomonas incerta & Reinhardtinia & linear & $\sim 17.5^{\mathrm{c}}$ & $\sim 75^{c}$ & $\sim 44^{c}$ & $\underline{\mathrm{DQ} 373068}$ & [35] \\
\hline Chlamydomonas reinhardtii & Reinhardtinia & linear & $15.8-18.9^{b}$ & $67-82$ & $\sim 45$ & $\begin{array}{l}\text { EU306617- } \\
\text { EU306623 }\end{array}$ & {$[29,30]$} \\
\hline Polytomella capuana & Reinhardtinia & linear & 13.0 & 82.0 & 57.2 & EF645804 & [36] \\
\hline Polytomella parva & Reinhardtinia & linear-fragmented & $16.2^{\mathrm{d}}$ & $65.5^{d}$ & $41.0^{\mathrm{d}}$ & $\begin{array}{l}\text { AY062933- } \\
\text { AY062934 }\end{array}$ & [33] \\
\hline Polytomella piriformisg & Reinhardtinia & linear-fragmented & $16.1^{d}$ & $65.8^{\mathrm{d}}$ & $42.0^{d}$ & $\begin{array}{l}\text { GU108480- } \\
\text { GU108481 }\end{array}$ & $N A$ \\
\hline Volvox carteri f. nagariensis & Reinhardtinia & circularf & $\sim 35 \mathrm{c}$ & $<40^{c}$ & $\sim 34^{c}$ & $\begin{array}{l}\text { EU760701, } \\
\text { GU084821 }\end{array}$ & {$[37,38]$} \\
\hline Chlamydomonas eugametos & Moewusinia & circular & 22.9 & 53.4 & 34.6 & $\underline{\mathrm{AF} 008237}$ & [31] \\
\hline Chlamydomonas moewusii & Moewusinia & circulare & $\sim 21 \mathrm{e}$ & -- & -- & -- & [39] \\
\hline Chlamydomonas pitschmannii & Moewusinia & circulare & $\sim 16.5^{\mathrm{e}}$ & -- & -- & -- & [40] \\
\hline Chlorogonium elongatum & Chlorogonia & circular & 22.7 & 53.3 & 37.8 & $\frac{Y 13643-Y 13644}{\underline{Y 07814}}$ & [32] \\
\hline Dunaliella salina & Dunaliellinia & circular & 28.3 & 42.0 & 34.4 & GQ250045 & this study \\
\hline \multicolumn{8}{|l|}{ PLASTID GENOMES } \\
\hline Chlamydomonas gelatinosa & Reinhardtinia & circulare & $\sim 285^{\mathrm{e}}$ & -- & -- & -- & [41] \\
\hline Chlamydomonas reinhardtii & Reinhardtinia & circular & 204.2 & 43.3 & 34.5 & $\underline{F J 423446}$ & [34] \\
\hline Volvox carteri f. nagariensis & Reinhardtinia & circular & $\sim 525^{c}$ & $<20^{c}$ & $\sim 43^{c}$ & $\underline{\text { GU084820 }}$ & {$[37,38]$} \\
\hline Chlamydomonas eugametos & Moewusinia & circulare & $\sim 243^{e}$ & -- & -- & -- & [39] \\
\hline Chlamydomonas moewusii & Moewusinia & circulare & $\sim 292^{\mathrm{e}}$ & -- & -- & -- & [39] \\
\hline Chlamydomonas pitschmannii & Moewusinia & circulare & $\sim 187^{e}$ & -- & -- & -- & [40] \\
\hline Dunaliella salina & Dunaliellinia & circular & 269.0 & 34.5 & 32.1 & $\underline{\mathrm{GQ} 250046}$ & this study \\
\hline $\begin{array}{l}\text { Note: "--", data not available. Cla } \\
\text { a Intronic open reading frames } \\
\text { b These data vary because of the } \\
\text { c These data are based on almos } \\
\text { d MtDNA consists of two fragme } \\
\text { e These data are based on gel-el } \\
\text { fThe circular conformation of th } \\
\text { 9 This strain is formally known a }\end{array}$ & $\begin{array}{l}\text { ined by Nakada } \\
\text { nsidered as codir } \\
\text { absence of optio } \\
\text { genome sequen } \\
\text { e based on the c } \\
\text { sis and Southern } \\
\text { ntDNA is based } \\
\text { a SAG 63-10. }\end{array}$ & $\begin{array}{l}\text { A.]. } \\
\text { A. } \\
\text { rons. } \\
\text { enation of these frag } \\
\text { analyses. } \\
\text { nome-assembly data }\end{array}$ & eec & & & & \\
\hline
\end{tabular}


Reinhardtinia clade have large, bloated plastid genomes. Both the C. reinhardtii and V. carteri plastid genomes, the only complete (or nearly complete) plastid DNA sequences that are available from the Chlamydomonadales, are among the largest and most noncoding-DNA dense plastid genomes observed to date, with sizes of 204 and $\sim 525$ kilobases (kb), respectively [34,38]. The forces driving these genomes towards distention are unknown, but they may be connected to the combined effects of a low mutation rate and a low effective population size [38].

Here we present the complete mitochondrial DNA (mtDNA) and plastid DNA (ptDNA) sequences of $D$. salina strain CCAP 19/18 -- a member of the Dunaliellinia clade [20]. The salient features of these genomes are described and compared with other chlamydomonadalean organelle genomes, particularly those of C. reinhardtii and V. carteri. The evolutionary and biotechnological implications of these sequences are discussed. The overreaching goals of this study are to use the D. salina organelle DNA data to test contemporary theories on genome evolution and to lay the foundation for a D. salina plastid transformation system.

\section{Results and discussion}

\section{Overview of the $D$. salina organelle genomes}

The organelle genome sequences of $D$. salina were assembled using publicly available trace files that the DOE JGI $D$. salina nuclear genome sequencing project produced (see the Methods section for a detailed description of how the genome assembly was performed). Genetic maps of the $D$. salina organelle genomes are shown in Figures 1 (mtDNA) and 2 (ptDNA); for comparison, these two figures also include the corresponding genetic maps from $C$. reinhardtii and Volvox carteri. Table 1 outlines the general features of the $D$. salina organelle genomes, including their length, coding and noncoding DNA contents and nucleotide compositions, and compares these statistics to those from other chlamydomonadalean organelle DNAs. A Venn diagram highlighting the differences in gene content among available mtDNA sequences from the Chlamydomonadales is presented in Figure 3. A schematic compilation comparing the amounts of noncoding DNA in the D. salina organelle genomes with those from other completely sequenced (and almost complete) organelle genomes is shown in Figure 4, and analyses of the repetitive elements within the D. salina organelle DNA are summarized in Figure 5 and Supplementary Figures S1 and S2 [see Additional files 1 and 2].

\section{Size, conformation, and nucleotide composition}

The D. salina mitochondrial and plastid genomes are 28.3 and $269 \mathrm{~kb}$, respectively, and assemble as circular mole- cules -- an observation that adds further support to the hypothesis that linear mitochondrial genomes in chlamydomonadalean algae are restricted to species within the Reinhardtinia clade (Table 1). The mitochondrial genome of D. salina is small relative to those of non-chlamydomonadalean green algae, which are, on average, 51.5 $\mathrm{kb}$; however, it is still 5.4-15.3 kb larger than all other available chlamydomonadalean mtDNAs, except for that of $V$. carteri, which is $\sim 35 \mathrm{~kb}([37,38])$. The size of the $D$. salina plastid genome is more pronounced than its mitochondrial counterpart, being the largest ptDNA sequenced thus far. Its nearest rivals are the $223.9 \mathrm{~kb}$ ptDNA of the chlorophycean green alga Stigeoclonium helveticum and the $217.9 \mathrm{~kb}$ plastid genome of the geranium Pelargonium $\times$ hortorum $[47,48]$. Large plastid genomes are a common theme among chlamydomonadalean algae: the $C$. reinhardtii plastid genome, at $204.2 \mathrm{~kb}$ [34], is the fourth largest completely sequenced ptDNA, partial sequence data indicate that the $V$. carteri ptDNA is $\sim 525 \mathrm{~kb}$ in length $([37,38])$, and gel electrophoresis results place the plastid genomes of Chlamydomonas gelatinosa (of the Reinhardtinia clade) and Chlamydomonas moewusii (of the Moewusinia clade [20]) at $\sim 285 \mathrm{~kb}$ and $\sim 292 \mathrm{~kb}$ in length, respectively $[39,41]$. The impressive size of the $D$. salina plastid genome (and those from other chlamydomonadalean algae) is a reflection of a prodigious noncoding DNA content rather than an unusually large gene repertoire. Within the D. salina ptDNA, a pair of inverted repeats, each with a length of $14.4 \mathrm{~kb}$, divides the genome into a large (127.3 kb) and a small single-copy region (112.9 kb), referred to as the LSC and SSC regions. The D. salina inverted repeats are $6.2 \mathrm{~kb}$ shorter than their C. reinhardtii counterparts. This size discrepancy occurs because the $C$. reinhardtii inverted repeats contain $p s b A$, a gene that is located in the SSC region of the $D$. salina ptDNA. Unlike in D. salina, the SSC and LSC regions of the $C$. reinhardtii ptDNA are virtually indistinguishable with sizes of $\sim 80 \mathrm{~kb}$. The precise lengths of the LSC, SSC, and inverted repeat regions for the $V$. carteri plastid genome are unknown; however, preliminary size estimates place them at $>25 \mathrm{~kb}$ [38]. Southern blot analyses and partial sequence data indicate that the inverted repeats of the C. moewusii ptDNA may be upwards of 40 $\mathrm{kb}$ [39].

The GC content of the $D$. salina organelle DNAs is $34.4 \%$ (mtDNA) and $32.1 \%$ (ptDNA), which is unremarkable in relation to other archaeplastidial organelle genomes (i.e., those from eukaryotes with primary plastids). However, they are still the most GC-poor (or ATrich) organelle DNAs observed within the Chlamydomonadales, which is significant because the Chlamydomonadales are one of the few lineages out of all eukaryotes known to contain species with GC-rich mito- 


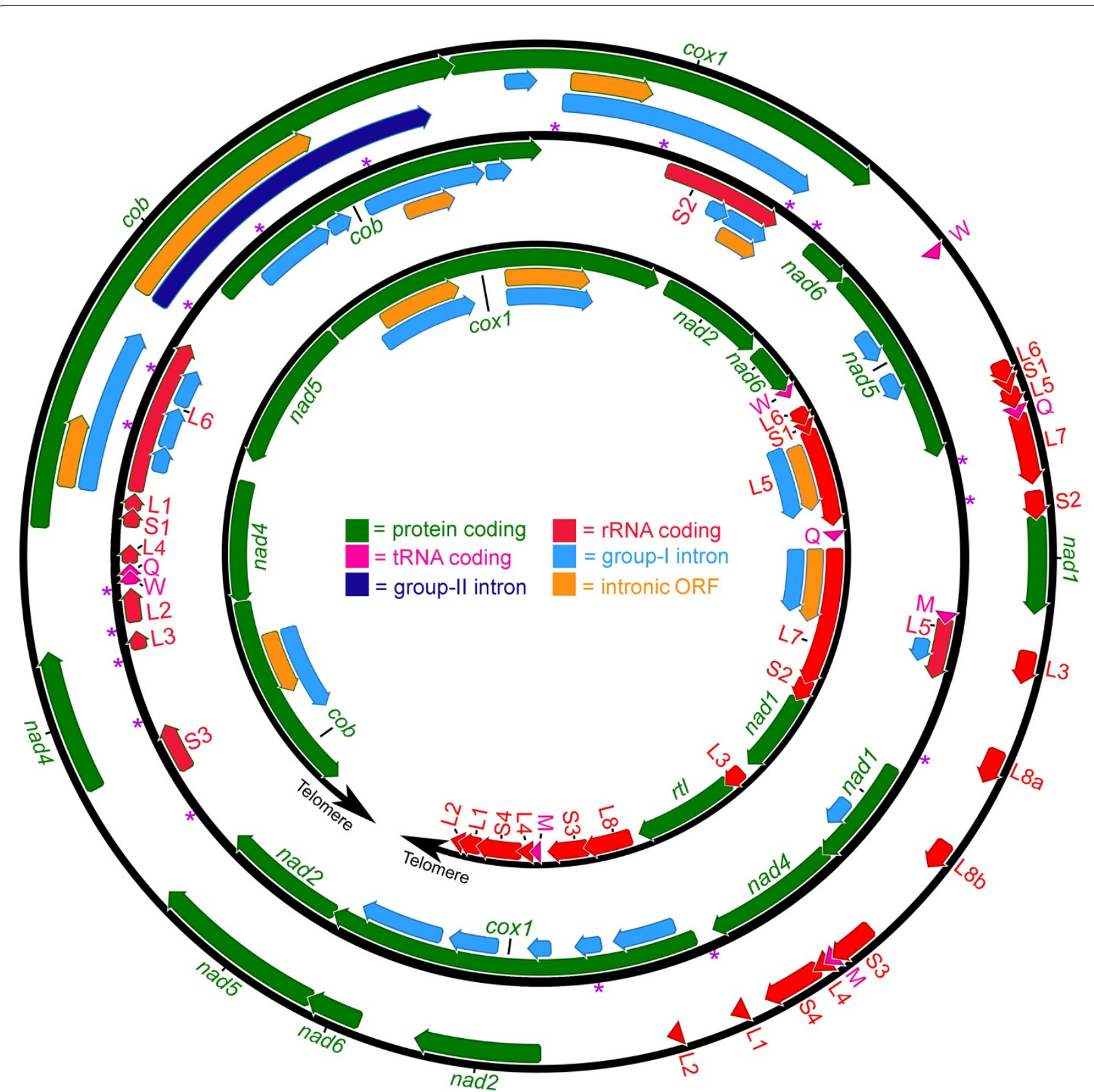

Figure 1 Complete mitochondrial genome maps for Dunaliella salina (middle), Chlamydomonas reinhardtii (inner), and Volvox carteri (outer). The mitochondrial genome of D. salina (this study) is $28.3 \mathrm{~kb}$, that of C. reinhardtii (GenBank accession numbers EU306617-EU306623) ranges from 15.8-18.9 kb, depending on the presence of optional introns, and that of $V$. carteri (GenBank accession numbers EU760701 and GU084821) is 35 kb. Note that the $C$. reinhardtii mtDNA is a linear molecule. Arrows within the coding regions denote transcriptional polarities. The small subunit and large subunit rRNA-coding regions are fragmented into modules. Transfer RNA-coding regions are designated by the single-letter abbreviation of the amino acid they specify. Purple asterisks denote the sites of palindromic repeat clusters (see Figure 5 for more details). Rt/ codes for a putative reversetranscriptase-like protein

chondrial genomes (Table 1) [36,43]. The GC content among the different regions of the D. salina mitochondrial and plastid genomes is relatively constant: $33 \%_{(m t D N A)}$ and $34 \%_{(p t D N A)}$ for coding DNA; $34 \%_{(m t D N A)}$ and $32 \%_{(p t D N A)}$ for introns and intronic open reading frames (ORFs); and $37 \%_{(m t D N A)}$ and $31 \%_{(p t D N A)}$ for intergenic regions. For the different codon-site positions of the mtDNA and ptDNA protein-coding regions, the GC content is approximately $38 \%_{(m t D N A)}$ and $42 \%_{(p t D N A)}\left(1^{\text {st }}\right.$ position); $38 \%_{(m t D N A)}$ and $52 \%_{(p t D N A)}\left(2^{\text {nd }}\right.$ position); and 
$19 \%_{(m t D N A)}$ and $13 \%_{(p t D N A)}\left(3^{\text {rd }}\right.$ position). Cumulative GCskew analyses, (often used to pinpoint origins of replication [49]) of the D. salina mtDNA show a strong positive correlation with the transcriptional orientation (data not shown), reflecting the slightly higher GC content of the coding and intronic regions relative to the noncoding mtDNA. The same analysis of the ptDNA gives a more disordered plot, but one typical of ptDNA, because of the many shifts in transcriptional polarity throughout the genome.

\section{Coding content}

Like other chlamydomonadalean species, $D$. salina has a severely diminished mtDNA gene content of only 12 genes, which represent seven proteins, two rRNAs, and three tRNAs. Outside of the Chlamydomonadales, the only species known to have more reduced mtDNA gene contents are found in the phyla Apicomplexa and Dinoflagellata and arguably some species within the supergroup Excavata [50,51]. Various studies have tried to explain why chlamydomonadalean algae have such reduced mtDNA gene contents [52,53], but at present no straightforward answer to this question exists. The $D$. salina mtDNA gene inventory mirrors those of Chlorogonium elongatum and Chlamydomonas eugametos, but it shows some differences to those of Reinhardtinia-clade algae. These differences, which can be visualized on the Venn diagram in Figure 3, involve changes in tRNA-coding content and in the number of rRNA-coding fragments found on the genome. For example, the $D$. salina mtDNA encodes three tRNAs, whereas Polytomella mtDNA contains only trnM, and the mitochondrial rrns and $r r n l$ genes of D. salina are divided into three (S1-S3) and six (L1-L6) coding modules, whereas in available Reinhardtinia-clade mtDNAs the rrns and $r r n l$ genes are fragmented into at least four and eight coding modules, respectively. Given the similarities among the D. salina, C. elongatum, and C. eugametos mitochondrial genomes, these findings add further appreciation for the stability of mtDNA gene content among chlamydomonadalean species outside of the Reinhardtinia clade and underscore the instability of mtDNA gene content among Reinhardtinia-clade taxa (Figure 3).

The $D$. salina plastid genome is much more gene rich than its mitochondrial counterpart, with 102 genes -- five of which are duplicates found in the inverted repeats. When ignoring these duplicates, there are a total of 66 protein-, 3 rRNA-, and 28 tRNA-coding genes. This gene content is reduced from those of green-plant species outside the Chlamydomonadales, which on average have 123 ptDNA-encoded genes, representing 85 proteins, 3 rRNAs, and 35 tRNAs. It appears that chlamydomonadalean algae, at some point during their evolution, went through a major reduction in ptDNA (and
mtDNA) coding content relative to most other photosynthetic eukaryotes. The $D$. salina ptDNA gene repertoire is identical to those of $C$. reinhardtii and V. carteri with the following minor exceptions: i) the $D$. salina plastid genome encodes three copies of trnI -- one more than the C. reinhardtii and V. carteri ptDNAs; ii) D. salina, like $C$. reinhardtii, has two ptDNA copies of trnE, whereas $V$. carteri has only one; iii) for $D$. salina and V. carteri, the rps 2 gene is represented by a single open reading frame, whereas for $C$. reinhardtii, rps 2 is fragmented into two adjacent open reading frames ( $r p s 2-a$ and $r p s 2-b$ ); and iv) the $D$. salina ptDNA does not contain the RoaA-like gene (orf494), which is present in the V. carteri ptDNA. Preliminary investigations of the plastids from Moewusiniaclade algae $[39,54]$ indicate that their ptDNA gene contents are similar to those of D. salina, C. reinhardtii, and $V$. carteri. Altogether, these findings suggest that ptDNA gene content is uniform throughout the Chlamydomonadales, save for some minor differences in the number of tRNA-coding genes.

\section{Gene order}

All 12 genes on the $D$. salina mitochondrial genome are encoded on the same strand (i.e., have identical transcriptional polarities), a characteristic shared by the three other circular-mapping chlamydomonadalean mtDNAs sequenced thus far (Table 1). Sequence data from chlamydomonadalean algae whose mtDNAs map as linear molecules, such as $C$. reinhardtii and Polytomella spp., reveal genomes that have two unequally sized gene clusters (i.e., a group of two or more genes that are situated close to one another) with opposing transcriptional polarities, which proceed outwards toward the ends of the genome (Figure 1). Based on these and the above observations, it is reasonable to assume that the ancestral chlamydomonadalean mtDNA mapped as a circular molecule with $\sim 12$ genes, all of which were encoded on the same strand, and that the events giving rise to linear mtDNA were connected with, or resulted in, a shift in transcriptional orientation of approximately one-third of the genes. The mtDNA gene order of $D$. salina is unique and differs from those of other chlamydomonadalean algae. Very few conserved mtDNA gene clusters are shared among $D$. salina and other chlamydomonadalean species (Figure 1), but this is not surprising considering that mtDNA gene arrangements can vary significantly even among closely related species in this group $[31,32,37,44]$. Previous reports suggest that homologous or illegitimate recombination between mtDNA repeats is causing mitochondrial genome rearrangements in chlamydomonadalean algae $[36,55,56]$. The $D$. salina mitochondrial genome does contain minor amounts of repetitive DNA (discussed below); these repeats may be catalysts for genome rearrangements. In C. reinhardtii, 


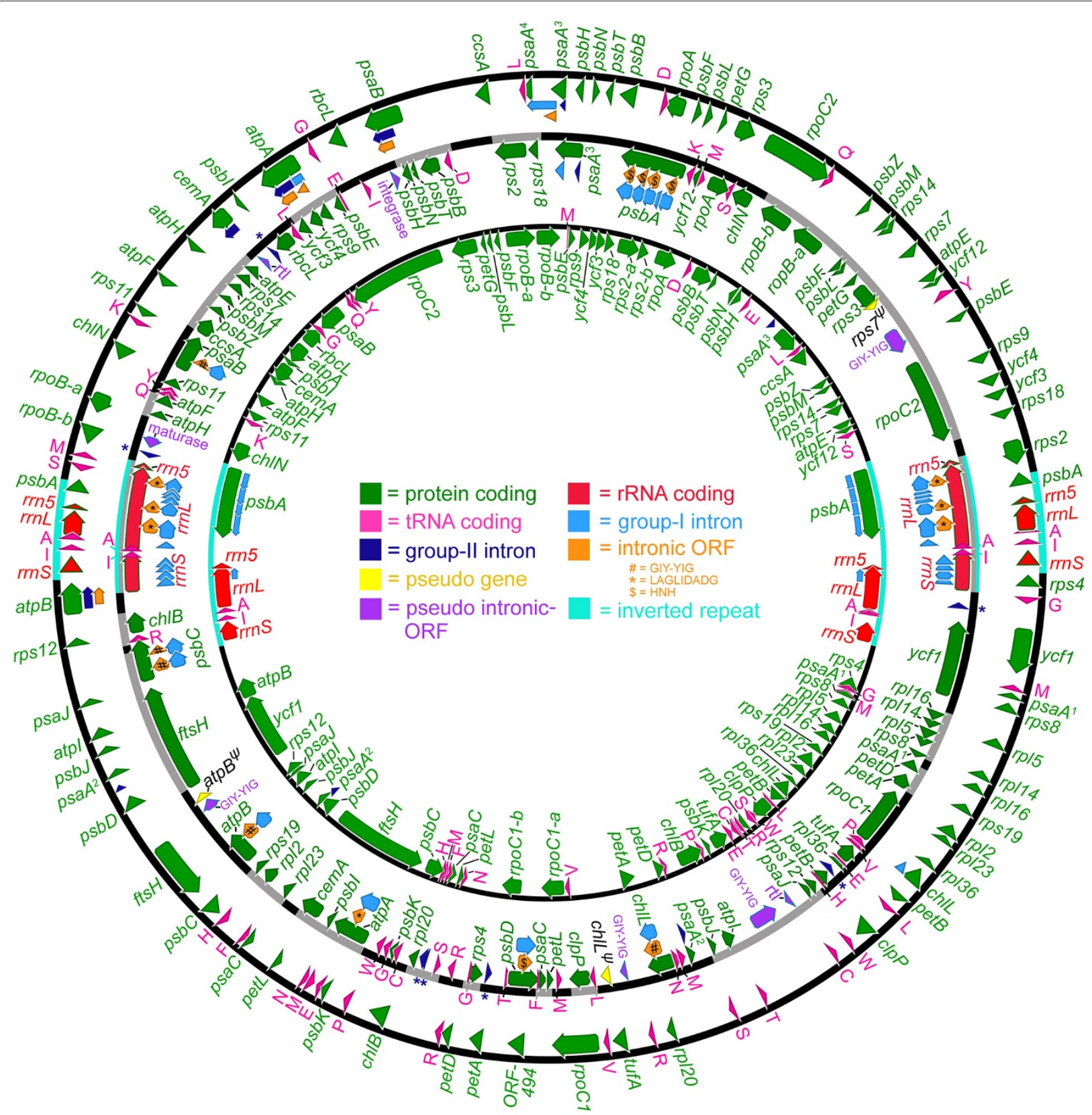

Figure 2 Complete plastid genome maps for Dunaliella salina (middle), Chlamydomonas reinhardtii (inner), and Volvox carteri (outer). The D. salina plastid genome (this study) is $269 \mathrm{~kb}$. The C. reinhardtii and V. carteri plastid genomes (GenBank accession numbers FJ423446 and GU084820) are $204.2 \mathrm{~kb}$ and $\sim 525 \mathrm{~kb}$, respectively. Arrows within the coding regions denote transcriptional polarities. Transfer RNA-coding regions are designated by the single-letter abbreviation of the amino acid they specify. Introns within intergenic regions are labeled with blue asterisks. Pseudogenes are labeled with a $\psi$. For all three genomes, the $p s a A$ gene is fragmented; the translational order of these fragments is set out using superscript numbers. The portions of the D. salina genome map that are gray (as opposed to black) highlight gene colinearity (not including introns) with either the C. reinhardtii or V. carteri plastid genomes.

mitochondrial genes are organized into operons, which are first transcribed into polycistronic primary transcripts and then subsequently processed into mature monocistronic units via endo- and exonucleolytic cleavage [29]. A scan of the D. salina mitochondrial genetic map reveals clusters of tightly packed genes separated by large stretches of noncoding DNA (Figure 1). These gene clusters may reflect the layout of operons in the genome, a theory supported by the fact that they are punctuated by regions of noncoding DNA that can be folded into secondary structures. 


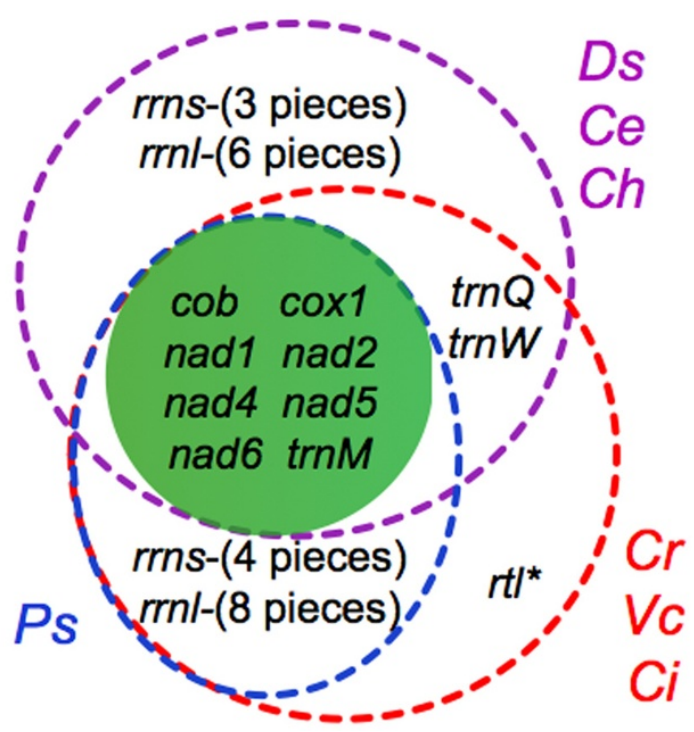

Figure 3 Venn diagram comparing the gene repertoires of chlamydomonadalean mitochondrial genomes. Chlamy-

domonadalean algae are labeled as follows: $\mathrm{Ce}=$ Chlamydomonas eugametos; $\mathrm{Ch}=$ Chlorogonium elongatum; $\mathrm{Ci}=$ Chlamydomonas incerta; $\mathrm{Cr}=$ Chlamydomonas reinhardtii; $\mathrm{Ds}=$ Dunaliella salina; $\mathrm{Ps}=$ Polytomella capuana, Polytomella parva, and Polytomella piriformis (strain SAG 63-10); Vc = Volvox carteri. * $R t /$ codes for a putative reverse-transcriptase-like protein: in C. reinhardtii and C. incerta this gene is independent of an intron, whereas in $\mathrm{V}$. carteri it is within a group-II intron. Note, the C. eugametos mtDNA contains a duplicate copy of trnM.

In contrast to the mtDNA, genes in the D. salina plastid genome are found on both strands and occur in small groups of two to four genes (Figure 2), which are distributed among the LSC and SSC regions and the inverted repeats (Figure 1). The former two regions contain approximately 50 and 45 genes, respectively, whereas the inverted repeats contain only five genes -- fewer than any chlamydomonadalean inverted repeat explored heretofore. The following additional genes are observed in the inverted repeats of other chlamydomonadalean algae: psbA (C. reinhardtii, V. carteri, C. moewusii, C. eugametos, C. gelatinosa, and Chlamydomonas pitschmannii), rbcL (C. moewusii and C. eugametos), and atpB (C. gelatinosa). Regions of gene synteny between the ptDNA of $D$. salina and those of $C$. reinhardtii and $V$. carteri (the only other chlamydomonadalean algae for which complete ptDNA maps are available) are highlighted in gray on Figure 2. The allocation of $D$. salina genes into small clusters is consistent with what is known for the $C$. reinhardtii plastid genome, where genes appear to be transcribed into monocistronic and dicistronic transcripts $[57,58]$ rather than the larger polycistronic transcripts that are observed for the mtDNA. Thus, regions of gene colinearity between $D$. salina and $C$. reinhardtii (or $V$. carteri) may represent conserved transcriptional units.

\section{Introns and intergenic regions}

One of the more salient features of the D. salina organelle genomes is their noncoding DNA content: $58 \%$ of the mtDNA and $65.5 \%$ of the ptDNA consist of either intergenic or intronic DNA. These values approach those of the $V$. carteri mitochondrial ( $>60 \%$ noncoding) and plastid (>80\% noncoding) genomes, which are currently the most inflated organelle DNA sequences from the Chlorophyta (a phylum containing most of the identified classes of green algae [26]) (Figure 4). In fact, next to $V$. carteri, the $D$. salina ptDNA has a greater noncoding DNA composition than any other plastid genome sequenced to date, exceeding that of the legume Trifolium subterraneum (57.9\%) and C. reinhardtii (56.7\%) (Figure 4) $[34,59]$. However, one would expect the unsequenced plastid genomes of C. gelatinosa and C. moewusii, based on their estimated sizes $[39,41]$, to have more noncoding DNA than $D$. salina but less than that of $V$. carteri (i.e., between 65-80\% noncoding). Interestingly, both the mitochondrial and plastid genomes of $D$. salina have equally large noncoding DNA densities (58\% vs. $65.5 \%$ ). This observation goes against what is seen in C. reinhardtii where the mtDNA and ptDNA have opposing architectures ( $\sim 20 \%$ vs. $\sim 57 \%$ noncoding), but it is consistent with the $V$. carteri organelle genomes, which are both distended with noncoding DNA (>60\%).

The noncoding DNA in the $D$. salina organelle genomes can be subdivided into two categories: intergenic regions, which make up $8.37 \mathrm{~kb}(29.5 \%)$ of the mtDNA and $139.65 \mathrm{~kb}(52 \%)$ of the ptDNA, and introns and intronic ORFs, which together represent $8.05 \mathrm{~kb}$ (28.5\%) and $36.49 \mathrm{~kb}(13.5 \%)$ of mitochondrial and plastid genomes, respectively. For the D. salina ptDNA, it is sometimes difficult to distinguish between intergenic DNA and intronic DNA because intron-like sequences (including intronic ORFs) are found in many of the intergenic regions (Figure 2). Altogether, 18 putative group-I introns were found in the mtDNA (two of which contain intronic ORFs) and 43 putative introns were discerned in the ptDNA: 36 within genes (35 of group-I and 1 of group-II affiliation) and 7 within intergenic regions (all of group-II affiliation). See Figures 1 and 2 as well as Supplementary Table 1 [Additional file 3] for a comparison of the organelle genome intron content of $D$. salina with those of $C$. reinhardtii and $V$. carteri. Note, because of the inverted repeats, 11 of the 43 introns in the ptDNA are duplicates (the single-copy-intron count for the ptDNA is 32). Seventeen of the gene-located ptDNA introns contain ORFs (their families are shown on Figure 2), whereas no ORFs were found within the ptDNA intergenic introns. The remnants of eight intronic ORFs (pseudo ORFs) were found in the intergenic ptDNA regions; these pseudo ORFs, which are often located adjacent to inter- 


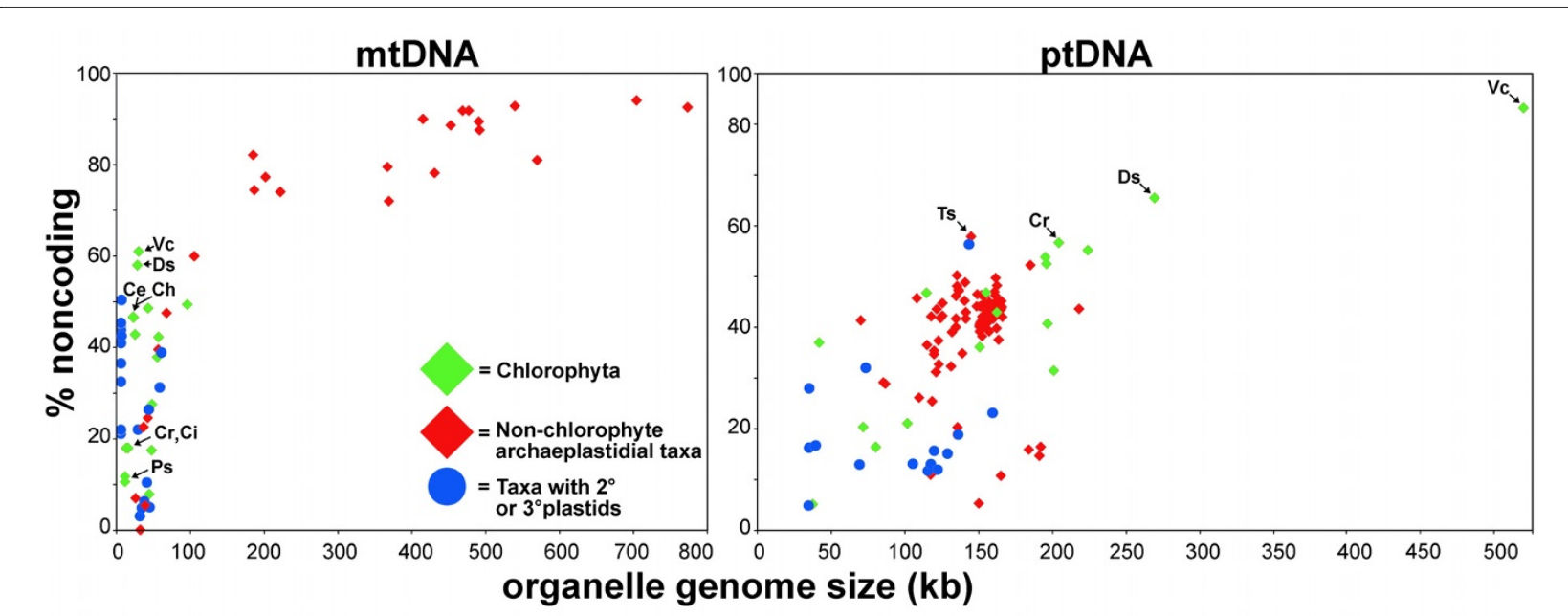

Figure 4 Scaling of noncoding DNA content with genome size in completely sequenced organelle DNAs. Chlamydomonadalean algae are labeled as follows: $\mathrm{Ce}=$ Chlamydomonas eugametos; $\mathrm{Ch}=$ Chlorogonium elongatum; $\mathrm{Ci}=$ Chlamydomonas incerta; $\mathrm{Cr}=$ Chlamydomonas reinhardtii; $\mathrm{Ds}$ = Dunaliella salina; PS = Polytomella capuana, Polytomella parva, and Polytomella piriformis (strain SAG 63-10); Ts = Trifolium subterraneum; Vc = Volvox carteri.

genic introns (Figure 2), appear to be nonfunctional because they contain frameshifts in their coding regions. All eight of the pseudo ORFs show sequence similarity to genes that are typically found in either group-I or groupII introns (Figure 2), such as genes coding for integrase-, maturase-, reverse-transcriptase- and endonuclease-like proteins. Intergenic introns have been identified in other genomes [60,61], but until now they had never been observed in chlamydomonadalean organelle DNA, or, to the best of our knowledge, any other green-algal organelle genomes. Most of the intergenic introns are highly derived and could only be identified using domain V, which is the most conserved secondary structure element of group-II introns [62]. Further experiments will need to be performed to confirm that the intergenic introns are functional (i.e., removed from mature transcripts) rather than inert sequences. If they are functional, then it would imply that many of the intergenic regions of the D. salina plastid genome are transcribed. There is also the possibility that the individual intergenic introns represent the fragments of larger introns that assemble after translation (i.e., the RNA fragments come together via base-pairing to form larger RNA species that are capable of splicing). However, secondary structure modeling of the intergenic introns gave no obvious indications that this was the case.

The intron/gene ratios for the D. salina mitochondrial and plastid genomes are 1.5 and 0.42 , respectively. These values are much larger than those of other chlamydomonadalean organelle genomes, which range from 0 to 0.75 for available mitochondrial genomes, and are less than 0.07 for the two available ptDNA sequences. Notably, the $D$. salina ptDNA intron/gene ratio exceeds the average value for land-plant mitochondrial genomes
( 0.6) [63], which are considered to be among the most intron-dense organelle DNAs.

The D. salina organelle DNAs contain significantly more introns than their $C$. reinhardtii and $V$. carteri counterparts (Figures 1 and 2). In stark contrast to the 18 introns found in the D. salina mtDNA, C. reinhardtii and $V$. carteri have five (all group I) and four (two group I and one group II) mitochondrial introns, respectively. Moreover, for $C$. reinhardtii all five introns are "optional" and, as of yet, the maximum number found in a single strain is three [52]. A similar trend is observed for the ptDNA: when counting duplicate genes only once, the plastid genome of $C$. reinhardtii has six introns (five group I and one group II) and that of $V$. carteri has eight (three group I and five group II); both these values are significantly less than the 32 unique putative introns found in the D. salina plastid genome. Interestingly, for all of the genes that contain introns in the C. reinhardtii and $V$. carteri organelle DNAs, their homologues in D. salina also contain introns, with the exception of $\operatorname{cem} A$, which is intronless in $D$. salina but contains a group-II intron in $V$. carteri. The number of introns per gene and the intron insertion sites can differ among these three algae.

Other notable features of the $D$. salina noncoding organelle DNA include three pseudogenes (rps $7 \psi, a t p B \psi$, and $\left.c h l L^{\psi}\right)$ in the plastid genome. These pseudogenes, whose functional copies are also present in the ptDNA, were classified as such, not because they contain frameshifts in their coding sequence or because they appear highly degenerate relative to their functional counterparts, but because they are missing the first half or first two-thirds of their coding sequences. Furthermore, atpB $\psi$ and $c h l L^{\psi}$ are located immediately downstream of their 


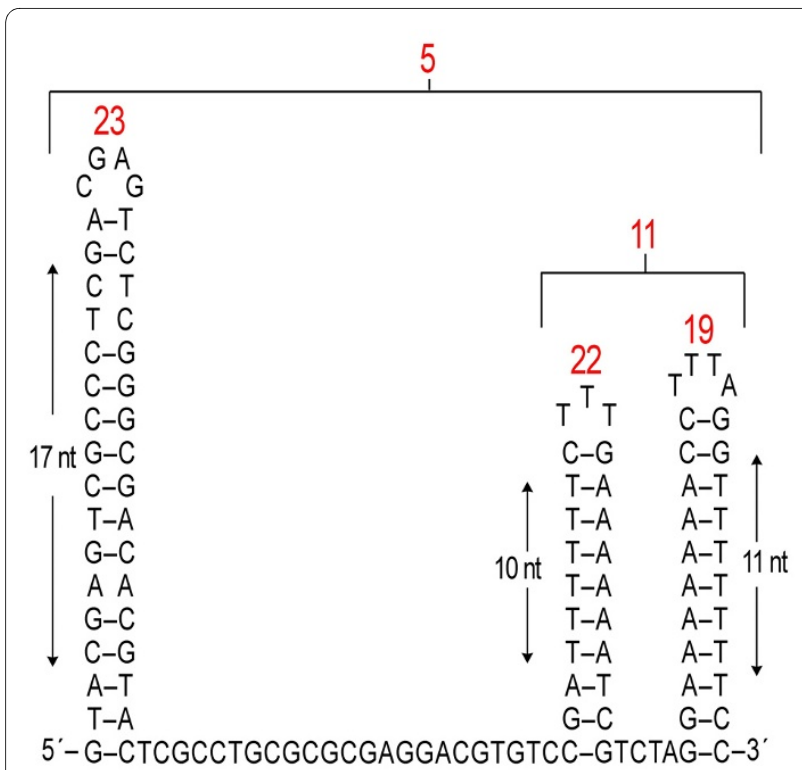

Figure 5 Consensus sequences and secondary structures of the D. salina mitochondrial palindromic repeat elements. The number of times each element appears in the $D$. salina mitochondrial genome is shown in red numbers. The locations of these palindromic elements within the mtDNA are depicted on Figure 1 using purple asterisks.

functional copies, and in both cases a group-I intron is sandwiched between the functional gene and the pseudogene (Figure 2).

\section{Repeats}

Unlike the mtDNA, which is relatively devoid of repeats, the $D$. salina plastid genome abounds with repetitive elements. The difference in repeat content between the mitochondrial and plastid genomes can be visualized by comparing their respective dotplot similarity matrices, which are shown in Supplementary Figures S1 (mtDNA) and S2 (ptDNA) [see Additional files 1 and 2]. Looking at the ptDNA dotplot, it is apparent that the ptDNA repeats are found in intergenic regions, introns, and in some of the longer protein-coding genes, such as fts $\mathrm{H}, \mathrm{rpoC} 2$, and $y c f 1$. Nucleotide BLAST analyses of the D. salina plastid genome indicate that there are upwards of 5,000 repeats in the ptDNA, forming approximately 100 repeat subclasses. With some exceptions, these repeats range from 30-60 nt in length and are 70-90\% AT. Some shorter (10$20 \mathrm{nt}) \mathrm{GC}$-rich repeat elements $(>50 \% \mathrm{GC})$ were also identified. The high degree of sequence similarity among the different ptDNA repeats is attributable to homopolymer runs rather than a recurring sequence motif. The $C$. reinhardtii and $V$. carteri plastid genomes are also rich in repetitive DNA and Southern blot analyses suggest that the C. gelatinosa ptDNA is as well $[34,37,41]$. Presumably, C. pitschmannii plastid genome, the smallest ptDNA observed from the Chlorophyceae $(\sim 187 \mathrm{~kb})$, contains fewer repeats than other chlamydomonadalean ptDNAs [40], implying a connection between repeat content and plastid genome size. In a general sense, the $D$. salina ptDNA repeats are analogous to the short dispersed repeats described for the C. reinhardtii ptDNA [34], but they lack the consistent motif of the palindromic elements of the $V$. carteri plastid genome [37].

The $D$. salina mtDNA dotplot reveals a mostly blank matrix, with the exception of some small diagonal lines, which correspond to palindromic repeats (i.e., repeats that can be folded into hairpin structures). Clusters of palindromic repeats are found in 18 different noncoding regions of the mitochondrial genome. The genomic breadth of these clusters can be seen in Figure 1, where purple asterisks pinpoint the precise location of these repeat clusters. A consensus sequence of one of the more frequently occurring clusters is depicted in Figure 5 . The mean length of the palindromic repeat clusters is $110 \mathrm{nt}$ and, on average, they have a GC content of $50 \%$. Each cluster contains approximately three palindromic elements (i.e., three putative hairpin structures), and the individual palindromes within each cluster range from 23-38 nt in length, and can be either AT- or GC-rich (Figure 5). Ten of the eighteen intergenic regions that are found in the mtDNA contain either one or two palindromic clusters (Figure 1), an arrangement that suggests that the palindromes may play a role in gene processing. Palindromic repeats have been described in other chlamydomonadalean mitochondrial genomes, including those of C. reinhardtii, V. carteri, and Polytomella capuana $[29,36,37,56]$, and in many cases chlamydomonadalean mtDNA palindromic repeats have been implicated in RNA processing $[36,37,64]$. A notable observation is that the mitochondrial palindromic repeats of both $D$. salina and $V$. carteri often contain the sequence 5'-TTTA-3' (or 5'-TTT-3') in the loops of their hairpin structures (Figure $5)$.

\section{Organelle DNA from other $D$. salina strains}

Prior to this study, organelle DNA sequence data for $D$. salina was limited to 14 GenBank entries amounting to $6.7 \mathrm{~kb}$ of ptDNA, divided over six protein-coding loci (no mtDNA sequence data were available). Most of these 14 entries do not list the strains of D. salina that were used for sequencing and only a few are associated with published articles. Alignments of the CCAP 19/18 ptDNA data generated here with the $D$. salina ptDNA data available at GenBank reveal a significant amount of sequence divergence: pairwise nucleotide diversity values varied from $2.4 \%$ to $17.6 \%$, which suggests that none of the $14 D$. salina entries are derived from strain CCAP 19/18 and that at least some of the sequences at GenBank labeled $D$. salina are not the same species as CCAP 19/18. In comparison, the average silent-site ptDNA diversity among 
seven different North American isolates of $C$. reinhardtii was estimated to be $1.4 \%$ [46], and a recent study on seven $V$. carteri geographical isolates found $\pi_{\text {silent }}$ of the ptDNA to be $\sim 0.065 \%$ [38]. The high degree of sequence diversity among $D$. salina GenBank entries appears to be a reflection of the known issue of misidentification of Dunaliella salina isolates. Dunaliella researchers should be mindful of the high levels of sequence divergence between CCAP 19/18 and the available D. salina GenBank entries if using these data for sequence-based studies, such as designing PCR primers.

\section{Paving the way towards plastid transformation}

The development of a reliable, high-efficiency genetic transformation system for $D$. salina is an important objective for the Dunaliella research community, especially when considering the many industrial applications that this technology could provide. Genetic transformation of the $D$. salina nuclear genome has been successful $[21,65]$; however, a lack of ptDNA sequence data has prevented successful attempts at transforming the $D$. salina plastid genome -- although an unsuccessful attempt was made to transform the plastid of Dunaliella tertiolecta [12]. For Dunaliella species, there are significant advantages to plastid transformation over nuclear transformation (see Verma and Daniell [14] for a review), some of which relate to the fact that ptDNA is polyploid and experiences high levels of gene expression. Now that the D. salina ptDNA sequence is available, scientists will be able to use these data to develop plastid transformation vectors targeting specific regions of the $D$. salina ptDNA. Plastid transformation occurs via homologous recombination between an engineered vector and a selected region of the plastid genome. Moreover, promoter and 3' UTR regions of genes [13] can now be used to design vectors for $D$. salina with improved expression levels. In principle, transgenes can be integrated into any site of the plastid genome, but transcriptionally-active intergenic regions are ideal [14]. One of the most frequently used integration sites is the trnI-trnA intergenic spacer, which is found in the inverted repeat of most plastid genomes, including that of $D$. salina. Given its popularity, the trnI$\operatorname{trn} A$ intergenic region would be an ideal site for early attempts at transforming the $D$. salina ptDNA. However, the discovery of intergenic introns in the $D$. salina ptDNA is an indication that many of the intergenic regions are transcriptionally active, which, if true, should allow for a diverse range of transformation targeting sites.

\section{Conclusion}

The $D$. salina organelle genomes are large, intron-dense molecules comprised predominantly of noncoding nucleotides. Repetitive elements punctuate the noncoding regions of the mitochondrial and plastid genomes, but are much more prevalent in the ptDNA. Overall, the dis- covery of putative intergenic introns in the $D$. salina ptDNA adds a new layer of complexity to the diverse repertoire of organelle genome architectures found in the Chlamydomonadales. Already a model organism for synthesizing $\beta$-carotene and studying salt adaptation, $D$. salina is now an ideal species for investigating organelle genome expansion. The high level of repetitive elements found in the plastid genome of D. salina may mirror the high level of repetitive sequences that is expected to be present in the nucDNA (DOE JGI, unpublished data). Publication of the plastid genome of $D$. salina is expected to result in major advances of plastid engineering with generation and use of transgenic D. salina strains for a number of new applications in the fields of biofuels as well as in vaccine antigene and biopharmaceutical production. The complete sequence of the $D$. salina nuclear genome will be made available soon, placing $D$. salina in a group of a select few photosynthetic eukaryotes for which complete genome sequences from all three genetic compartments are available.

\section{Methods}

\section{D. salina strain information}

The organelle DNA sequence data presented in this study come from D. salina strain CCAP 19/18, which is maintained at the Culture Collection of Algae and Protozoa (CCAP) in Argyll, Scotland [66]. D. salina CCAP 19/18 originates from the hypersaline Hutt Lagoon in Western Australia.

\section{Assembly of the D. salina organelle-genome sequences}

The complete mitochondrial and plastid genome sequences of $D$. salina were generated by collecting and assembling the publicly available mtDNA and ptDNA trace files that the DOE JGI $D$. salina nuclear genome sequencing project produced [22]. Trace files were datamined from the National Center for Biotechnology Information (NCBI) D. salina Trace Archive [24] using the following complete organelle genome sequences as trace BLAST (blastn 2.2.21+) queries: the C. elongatum and $C$. eugametos mitochondrial genomes, and the $C$. reinhardtii and $V$. carteri mtDNAs and ptDNAs -- similar approaches to assembling organelle genomes have been used in other studies (e.g., Smith and Lee [46]; Voigt et al. [67]). The BLAST parameters were as follows: an expectation value (E-value) of 10; a word size of 11; match and mismatch scores of 2 and -3, respectively; and gap-cost values of 5 (existence) and 2 (extension). Trace files showing $>80 \%$ sequence similarity to the BLAST queries were downloaded and then assembled with CodonCode Aligner Version 2.0.6 (CodonCode Corporation, Dedham, MA, USA), which employs the Phred, Cross-match, and Phrap algorithms for base calling, sequence comparison, and sequence assembly, respectively. Assemblies were performed with a minimum percent identity score 
of 98 , a minimum overlap length of $500 \mathrm{nt}$, a match score of 1 , a mismatch penalty of -2 , a gap penalty of -2 , and an additional first gap penalty of -3 . Gaps in the assemblies were filled by trace file walking, which was carried out by using $D$. salina mtDNA and ptDNA trace files as BLAST queries against the $D$. salina trace archive -- a process that allows one to "walk" slowly in both directions along the contigs, thereby, filling in any gaps. The final assemblies of the D. salina mtDNA and ptDNA trace files gave complete mitochondrial and plastid genome sequences with greater than 50 -fold coverage.

\section{Analyses of introns and repetitive DNA in the D. salina organelle genomes}

Introns in the D. salina organelle genomes were detected, classified, and folded into secondary structures using RNAweasel [62] and Rfam [68]. Introns that were not detected by these programs were identified by their ability to be folded into suitable secondary structures. Intron/gene ratios were calculated by dividing the number of introns in the genome by the gene number; for the D. salina ptDNA, intergenic introns were included in this calculation. Dotplot similarity matrices were generated with JDotter (version 12.2.0) using a sliding window size of 50 [69]. Mfold [70] was employed for all secondary structure analyses.

The D. salina organelle-DNA sequences were initially scanned for repeats with REPuter [71] using the Hamming distance option and a minimal repeat size setting of 12 nt. Forward, reverse, complement, and reverse complement repeats were all considered under REPuter. More detailed analyses of the $D$. salina organelle genomes for repeats were performed by building a custom BLAST databank of the mtDNA and ptDNA sequences and then comparing (blastn version 2.2.21+) this databank with specific regions from the mitochondrial and plastid genomes using an E-value of 5, a word size of 7, a match score of 2 , a mismatch penalty of -3 , a gap open score of 5 , and a extend value of 2 .

\section{The fraction of noncoding DNA in completely sequenced organelle genomes}

Completely sequenced organelle genomes were downloaded from the NCBI Reference Sequence (RefSeq) collection [72] on June 1, 2009. The coding and noncoding DNA contents of these sequences were calculated using the following methods and definitions: i) the number of coding nucleotides in the genome is equal to the collective length of all annotated protein-, rRNA-, and tRNAcoding regions -- not including the portions of these regions that are also annotated as introns; ii) the amount of noncoding DNA is the genome length minus the number of coding nucleotides; iii) the number of intergenic nucleotides is equal to the genome length minus the collective length of regions annotated as genes (including their introns and intronic ORFs); and iv) the amount of intronic DNA is equivalent to the number of noncoding nucleotides minus the number of intergenic nucleotides. The above methods and definitions are contingent on the authors of the GenBank records having properly annotated their entry. If coding regions or introns have been ignored or inaccurately annotated, coding and noncoding DNA content values will be incorrect. All records were quickly scanned for major errors, but due to the large number of organelle genomes deposited in GenBank, it was unfeasible to review all of the records thoroughly.

\section{GenBank accession numbers}

The GenBank accession numbers of the D. salina organelle-genome sequences are GQ250045 (mtDNA) and GQ250046 (ptDNA).

\section{Additional material}

\section{Additional file 1 Figure S1. Dotplot similarity matrix of the D. salina mito- chondrial genome. \\ Additional file $\mathbf{2}$ Figure S2. Dotplot similarity matrix of the $D$. salina plas tid genome. \\ Additional file $\mathbf{3}$ Table S1. Intron content of the D. salina, C. reinhardtii, and $V$. carteri organelle genomes.}

\section{Authors' contributions}

DRS analyzed the data and wrote the manuscript. RWL, JCC, JKM and JEP helped in interpreting the data and revising the manuscript. DT cultivated the D. salina, isolated the DNA, and helped annotate the genomes. JCC provided the nucleic acids used for organellar and genomic sequencing. JCC, JKM, and JEP are the external leaders of the DOE JGI Dunaliella salina CCAP 19/18 nuclear genome sequencing project. All authors have read and approved the final manuscript.

\section{Acknowledgements}

This work was supported by a grant to RWL from the Natural Sciences and Engineering Research Council (NSERC) of Canada. DRS. is an Izaak Walton Killam Memorial Scholar and holds a Canada Graduate Scholarship from NSERC. Additional funding was provided by a SunGrant Initiative grant awarded to JCC. We thank Leyla Hathwaik for technical assistance and the DOE JGI for sequencing the $D$. salina genome and providing the data for this study. Support for the Nevada Genomics, Proteomics, and Bioinformatics Centers was made possible by NIH Grant Number P20 RR-016464 from the INBRE-BRIN Program of the National Center for Research Resources and the NIH IDeA Network of Biomedical Research Excellence (INBRE, RR-03-008). The work conducted by the DOE JGI is supported by the Office of Science of the U.S. Department of Energy under Contract No. DE-AC02-05CH11231.

\section{Author Details}

1Department of Biology, Dalhousie University, Halifax, NS, B3H 4J1, Canada, 2Department of Biochemistry and Molecular Biology, MS200, 311B Fleischmann Agriculture, University of Nevada, Reno, NV 89557-0014, USA, ${ }^{3}$ Chemical and Biological Process Development, Energy and Environment Directorate, Pacific Northwest National Laboratory, 902 Battelle Blvd, Richland, WA 99352, USA and ${ }^{4}$ Department of Biology, Brooklyn College of the City University of New York, 2900 Bedford Ave, 200 NE, Brooklyn, NY 11210, USA

Received: 30 November 2009 Accepted: 7 May 2010

Published: 7 May 2010 


\section{References}

1. Teodoresco EC: Organisation et développement du Dunaliella, nouveau genre de Volvocacée-Polyblépharidée. Beih Bot Zentralblatt Bd 18 Abt 1905, 1:215-232

2. Oren A: A hundred years of Dunaliella research: 1905-2005. Saline Syst 2005, 1:2.

3. Polle JEW, Tran D, Ben-Amotz A: History, distribution, and habitats of algae of the genus Dunaliella Teodoresco (Chlorophyceae). In The Alga Dunaliella: Biodiversity, Physiology, Genomics, and Biotechnology Edited by: Ben-Amotz A, Polle JEW, Rao DVS. Enfield, New Hampshire, USA: Science Publishers: 2009:1-14.

4. Tafresh AH, Shariati M: Dunaliella biotechnology: methods and applications. J App/Microbio/ 2009, 107:14-35.

5. Ben-Amotz A, Katz A, Avron M: Accumulation of $\beta$-carotene in halotolerant algae: Purification and characterization of $\beta$-carotene-rich globules from Dunaliella bardawil (Chlorophyceae). J Phycol 1982, 18:529-537

6. Katz A, Jiménez C, Pick U: Isolation and characterization of a protein associated with carotene globules in the alga Dunaliella bardawil. Plant Physiol 1995, 108:1657-1664.

7. Moulton TP, Borowitzka LJ, Vincent DJ: The mass culture of Dunaliella salina for $\beta$-carotene: from pilot plant to production plant. Hydrobiologia 1987, 151/152:99-105.

8. Ben-Amotz A: Industrial production of microalgal cell-mass and secondary products - major industrial species. In Handbook of Microalgal Culture: Biotechnology and Applied Phycology Edited by: Richmond A. Oxford, UK: Blackwell Publishing; 2003:273-280.

9. Ben-Amotz A, Polle JEW, Rao DVS: The Alga Dunaliella: Biodiversity, Physiology, Genomics, and Biotechnology Enfield, New Hampshire, USA: Science Publishers; 2009

10. Avron M, Ben-Amotz A: Dunaliella: Physiology, Biochemistry, and Biotechnology. Boca Raton, Florida, USA: CRC Press Inc; 1992.

11. Bock R, Khan MS: Taming plastids for a green future. Trends in Biotechnology 2004, 22:311-318

12. Walker TL, Black D, Becker DK, Dale JL, Collet C: Isolation and characterization of components of the Dunaliella tertiolecta chloroplast genome. J Appl Phycol 2005, 17:495-508

13. Fletcher SP, Muto M, Mayfield SP: Optimization of recombinant protein expression in the chloroplasts of green algae. Adv Exp Med Biol 2007 616:90-98.

14. Verma D, Daniell H: Chloroplast vector systems for biotechnology applications. Plant Physio/ 2007, 145:1129-1143.

15. Purton S: Tools and techniques for chloroplast transformation of Chlamydomonas. Adv Exp Med Biol 2007, 616:34-45.

16. Rosenberg JN, Oyler GA, Wilkinson L, Betenbaugh MJ: A green light for engineered algae: redirecting metabolism to fuel a biotechnology revolution. Curr Opin in Biotech 2008, 19:430-436.

17. Boynton JE, Gillham NW, Harris EH, Hosler JP, Johnson AM, Jones AR, Randolph-Anderson BL, Robertson D, Klein TM, Shark KB, et al.: Chloroplast transformation in Chlamydomonas with high velocity microprojectiles. Science 1988, 240:1534-1538.

18. Goldschmidt-Clermont M: Transgenic expression of aminoglycoside adenine transferase in the chloroplast: a selectable marker for sitedirected transformation of Chlamydomonas. Nucleic Acids Res 1991, 19:4083-4089.

19. Maliga P: Plastid transformation in higher plants. Annu Rev Plant Biol 2004, 55:289-313.

20. Nakada T, Misawa K, Nozaki H: Molecular systematics of Volvocales (Chlorophyceae, Chlorophyta) based on exhaustive 18S rRNA phylogenetic analyses. Mol Phylogenet Evol 2008, 48:281-291.

21. Polle JEW, Qin S: Development of genetics and molecular tool kits for species of the unicellular green alga Dunaliella (Chlorophyta). In The Alga Dunaliella: Biodiversity, Physiology, Genomics, and Biotechnology Edited by: Ben-Amotz A, Polle JEW, Rao DVS. Enfield, New Hampshire, USA: Science Publishers; 2009:403-422.

22. United States Department of Energy Joint Genome Institute [http:// www.jgi.doe.gov/

23. Gouveia $L$, Oliveira AC: Microalgae as a raw material for biofuels production. J Ind Microbiol Biot 2009, 36:269-274.

24. NCBI Trace Archives [http://www.ncbi.nlm.nih.gov/Traces/home/

25. González MA, Gómez PI, JEW Polle: Taxonomy and phylogeny of the genus Dunaliella. In The Alga Dunaliella: Biodiversity, Physiology, Genomics, and Biotechnology Edited by: Ben-Amotz A, Polle JEW, Rao DVS. Enfield New Hampshire, USA: Science Publishers; 2009:15-44.

26. Lewis $L A, M c C$ Curt M: Green algae and the origin of land plants. Am J Bot 2004, 91:1535-1556.

27. Borowitzka MA, Borowitzka LJ: Dunaliella. In Microalgal Biotechnology Edited by: Borowitzka MA, Borowitzka LJ. Cambridge, UK: Cambridge University Press; 1988:27-58.

28. Borowitzka MA, Siva C: The taxonomy of the genus Dunaliella (Chlorophyta, Dunaliellales) with emphasis on the marine and halophilic species. J App/ Phycol 2007, 19:567-590.

29. Gray MW, Boer PH: Organization and expression of algal (Chlamydomonas reinhardtii) mitochondrial DNA. Philos TRoy Soc B 1988, 319:135-147.

30. Michaelis G, Vahrenholz C, Pratje E: Mitochondrial DNA of Chlamydomonas reinhardtii: the gene for apocytochrome $b$ and the complete functional map of the 15.8 kb DNA. Mol Gen Genet 1990 223:211-216.

31. Denovan-Wright EM, Nedelcu AM, Lee RW: Complete sequence of the mitochondrial DNA of Chlamydomonas eugametos. Plant Mol Biol 1998, 36:285-295.

32. Kroymann J, Zetsche K: The mitochondrial genome of Chlorogonium elongatum inferred from the complete sequence. J Mol Evol 1998 47:431-440

33. Fan J, Lee RW: Mitochondrial genome of the colorless green alga Polytomella parva: two linear DNA molecules with homologous inverted repeat termini. Mol Biol Evol 2002, 19:999-1007.

34. Maul JE, Lilly JW, Cui L, dePamphilis CW, Miller W, Harris EH, Stern DB: The Chlamydomonas reinhardtii plastid chromosome: islands of genes in a sea of repeats. Plant Cell 2002, 14:2659-2679.

35. Popescu CE, Lee RW: Mitochondrial genome sequence evolution in Chlamydomonas. Genetics 2007, 175:819-826.

36. Smith DR, Lee RW: Mitochondrial genome of the colorless green alga Polytomella capuana: a linear molecule with an unprecedented GC content. Mol Biol Evol 2008, 25:487-496.

37. Smith DR, Lee RW: The mitochondrial and plastid genomes of Volvox carteri: bloated molecules rich in repetitive DNA. BMC Genomics 2009 10:132

38. Smith DR, Lee RW: Low nucleotide diversity for the expanded organelle and nuclear genomes of Volvox carteri supports the mutational-hazard hypothesis. Mol Biol Evol in press.

39. Boudreau E, Otis C, Turmel M: Conserved gene clusters in the highly rearranged chloroplast genomes of Chlamydomonas moewusii and Chlamydomonas reinhardtii. Plant Mol Biol 1994, 24:585-602.

40. Boudreau E, Turmel M: Gene rearrangements in Chlamydomonas chloroplast DNAs are accounted for by inversions and by the expansion/contraction of the inverted repeat. Plant Mol Biol 1995 27:351-364

41. Boudreau E, Turmel M: Extensive gene rearrangements in the chloroplast DNAs of Chlamydomonas species featuring multiple dispersed repeats. Mol Biol Evol 1996, 13:233-243.

42. Vahrenholz C, Riemen G, Pratje E, Dujon B, Michaelis G: Mitochondrial DNA of Chlamydomonas reinhardtii: the structure of the ends of the linear 15.8-kb genome suggests mechanisms for DNA replication. Curr Genet 1993, 24:241-247.

43. Borza T, Redmond EK, Laflamme M, Lee RW: Mitochondrial DNA in the Oogamochlamys clade (Chlorophyceae): high GC content and unique genome architecture for green algae. J Phycol 2009, 45:1323-1334

44. Mallet M, Lee RW: Identification of three distinct Polytomella lineages based on mitochondrial DNA features. J Eukaryot Microbiol 2006 53:79-84.

45. Nedelcu AM: Contrasting mitochondrial genome organizations and sequence affiliations among green algae: potential factors, mechanisms, and evolutionary scenarios. J Phycol 1998, 34:16-28.

46. Smith DR, Lee RW: Nucleotide diversity of the Chlamydomonas reinhardtii plastid genome: addressing the mutational-hazard hypothesis. BMC Evol Biol 2009, 9:120.

47. Bélanger AS, Brouard JS, Charlesbois P, Otis C, Lemieux C, Turmel M: Distinctive architecture of the chloroplast genome in the chlorophycean green alga Stigeoclonium helveticum. Mol Genet Genomics 2006, 276:464-477

48. Chumley TW, Palmer JD, Mower JP, Fourcade HM, Calie PJ, Boore JL, Jansen RK: The complete chloroplast genome sequence of Pelargonium 
$\times$ hortorum: organization and evolution of the largest and most highly rearranged chloroplast genome of land plants. Mol Biol Evol 2006, 23:2175-2190.

49. Grigoriev A: Analyzing genomes with cumulative skew diagrams. Nucleic Acids Res 1998, 26:2286-2290.

50. Kairo A, Fairlamb AH, Gobright E, Nene V: A 7.1 kb linear DNA molecule of Theileria parva has scrambled rDNA sequences and open reading frames for mitochondrially endcoded proteins. EMBO J 1994, 13:898-905

51. Nash EA, Barbrook AC, Edwards-Stuart RK, Bernhardt K, Howe CJ, Nisbet RER: Organization of the mitochondrial genome in the dinoflagellate Amphidinium carterae. Mol Biol Evol 2007, 24:1528-1536.

52. Smith DR, Lee RW: Nucleotide diversity in the mitochondrial and nuclear compartments of Chlamydomonas reinhardtii: investigating the origins of genome architecture. BMC Evol Biol 2008, 8:156.

53. Lynch M, Koskella B, Schaack S: Mutation pressure and the evolution of organelle genomic architecture. Science 2006, 311:1727-1730.

54. Turmel M, Bellemare G, Lemieux C: Physical mapping of differences between the chloroplast DNAs of the interfertile algae Chlamydomonas eugametos and Chlamydomonas moewusii. Curr Genet 1987, 11:543-552.

55. Nedelcu AM: Fragmented and scrambled mitochondrial ribosomal RNA coding regions among green algae: a model for their origin and evolution. Mol Biol Evol 1997, 14:506-517.

56. Nedelcu AM, Lee RW: Short repetitive sequences in green algal mitochondrial genomes: potential roles in mitochondrial genome evolution. Mol Biol Evol 1998, 15:690-701.

57. Sakamoto W, Sturm NR, Kindle KL, Stern DB: petD mRNA maturation in Chlamydomonas reinhardtii chloroplasts: role of 5 ' endonucleolytic processing. Mol Cell Biol 1994, 14:6180-6186.

58. Jiao HS, Hicks A, Simpson C, Stern DB: Short dispersed repeats in the Chlamydomonas chloroplast genome are collocated with sites for mRNA 3' end formation. Curr Genet 2004, 45:311-322.

59. Cai Z, Guisinger M, Kim HG, Ruck E, Blazier JC, McMurty V, Kuehl JV, Boore $J$, Jansen RK: Extensive reorganization of the plastid genome of Trifolium subterraneum (Fabaceae) is associated with numerous repeated sequences and novel DNA insertions. J Mol Evol 2008 , 67:696-704.

60. Simon DM, Clark N, McNeil BA, Johnson I, Pantuso D, Dai L, Chai D, Zimmerly S: Group II introns in Eubacteria and Archaea: ORF-less introns and new varieties. RNA 2008, 14:1704-1713.

61. Tourasse NJ, Kolst $\varnothing$ AB: Survey of group I and group II introns in 29 sequenced genomes of the Bacillus cereus group: insights into their spread and evolution. Nucleic Acids Res 2008, 14:4529-4548.

62. Lang BF, Laforest MJ, Burger G: Mitochondrial introns: a critical view. Trends Genet 2007, 23:119-125.

63. Lynch M: The Origins of Genome Architecture Sunderland: Sinauer Associates, Inc; 2007

64. Boer PH, Gray MW: Short dispersed repeats localized in spacer regions of Chlamydomonas reinhardtii mitochondrial DNA. Curr Genet 1991, 19:309-312.

65. Feng S, Xue L, Liu H, Lu P: Improvement of efficiency of genetic transformation for Dunaliella salina by glass beads method. Mol Biol Rep 2009, 36:1433-1439.

66. Culture Collection of Algae and Protozoa [http://www.ccap.ac.uk/

67. Voigt $\mathrm{O}$, Erpenbeck $\mathrm{D}$, Wörheide $\mathrm{G}$ : A fragmented metazoan organellar genome: the two mitochondrial chromosomes of Hydra magnipapillata. BMC Genomics 2008, 9:350.

68. Griffiths-Jones S, Bateman A, Marshall M, Khanna A, Eddy SR: Rfam: an RNA family database. Nucleic Acids Res 2003, 31:439-441.

69. Brodie R, Roper RL, Upton C: JDotter: a java interface to multiple dotplots generated by dotter. Bioinformatics 2004, 20:279-281.

70. Zuker M: Mfold web server for nucleic acid folding and hybridization prediction. Nucleic Acids Res 2003, 31:3406-3415.

71. Kurtz S, Schleiermacher C: REPuter: fast computation of maximal repeats in complete genomes. Bioinformatics 1999, 15:426-427.

72. NCBI Reference Sequence (RefSeq) collection [http:// www.ncbi.nlm.nih.gov/projects/RefSeq/

doi: $10.1186 / 1471-2229-10-83$

Cite this article as: Smith et al., The Dunaliella salina organelle genomes: large sequences, inflated with intronic and intergenic DNA BMC Plant Biology 2010, 10:83

\section{Submit your next manuscript to BioMed Central} and take full advantage of:

- Convenient online submission

- Thorough peer review

- No space constraints or color figure charges

- Immediate publication on acceptance

- Inclusion in PubMed, CAS, Scopus and Google Scholar

- Research which is freely available for redistribution 